\title{
Patterns of Multimorbidity in a Population-Based Cohort of Older People: Sociodemographic, Lifestyle, Clinical, and Functional Differences
}

\author{
Alessandra Marengoni, PhD, ${ }^{1,2, *,+}$ Albert Roso-Llorach, MSc, ${ }^{3,4,+}$ Davide L. Vetrano, MD, \\ PhD, ${ }^{1,5,6}$ Sergio Fernández-Bertolín, MSc, ${ }^{3,4}$ Marina Guisado-Clavero, MD, ${ }^{3,4}$ Concepción \\ Violán, $\mathrm{PhD}^{3,4}$ and Amaia Calderón-Larrañaga, $\mathrm{PhD}^{1}$
}

'Aging Research Center, Department of Neurobiology, Care Sciences and Society, Karolinska Institutet and Stockholm University, Sweden. ${ }^{2}$ Department of Clinical and Experimental Sciences, University of Brescia, Italy. ${ }^{3}$ Fundació Institut Universitari per a la recerca a I'Atenció Primària de Salut Jordi Gol i Gurina (IDIAPJGol), Spain. ${ }^{4}$ Universitat Autònoma de Barcelona, Bellaterra (Cerdanyola del Vallès), Spain. ${ }^{5}$ Department of Geriatrics, Catholic University of Rome, Italy. ${ }^{6}$ Centro di Medicina dell'Invecchiamento, Fondazione Policlinico “A. Gemelli," Scientific Institute for Research and Healthcare (IRCCS), Rome, Italy.

${ }^{*}$ Address correspondence to: Alessandra Marengoni, PhD, Department of Clinical and Experimental Sciences, University of Brescia, Viale Europa 11, 25123 Brescia, Italy. E-mail: alessandra.marengoni@unibs.it

${ }^{\dagger}$ First shared authorship.

Received: February 28, 2019; Editorial Decision Date: May 20, 2019

Decision Editor: Anne Newman, MD, MPH

\begin{abstract}
Background: The aim of this study is to identify clusters of older persons based on their multimorbidity patterns and to analyze differences among clusters according to sociodemographic, lifestyle, clinical, and functional characteristics.

Methods: We analyzed data from the Swedish National Study on Aging and Care in Kungsholmen on 2,931 participants aged 60 years and older who had at least two chronic diseases. Participants were clustered by the fuzzy $c$-means cluster algorithm. A disease was considered to be associated with a given cluster when the observed/expected ratio was $\geq 2$ or the exclusivity was $\geq 25 \%$.

Results: Around half of the participants could be classified into five clinically meaningful clusters: respiratory and musculoskeletal diseases (RESP-MSK) $15.7 \%$, eye diseases and cancer (EYE-CANCER) 10.7\%, cognitive and sensory impairment (CNS-IMP) $10.6 \%$, heart diseases (HEART) $9.3 \%$, and psychiatric and respiratory diseases (PSY-RESP) $5.4 \%$. Individuals in the CNS-IMP cluster were the oldest, with the worst function and more likely to live in a nursing home; those in the HEART cluster had the highest number of co-occurring diseases and drugs, and they exhibited the highest mean values of serum creatinine and C-reactive protein. The PSY-RESP cluster was associated with higher levels of alcoholism and neuroticism. The other half of the cohort was grouped in an unspecific cluster, which was characterized by gathering the youngest individuals, with the lowest number of co-occurring diseases, and the best functional and cognitive status.

Conclusions: The identified multimorbidity patterns provide insight for setting targets for secondary and tertiary preventative interventions and for designing care pathways for multimorbid older people.
\end{abstract}

Keywords: Multimorbidity pattern, Older adults, Swedish National Study on Aging and Care in Kungsholmen (SNAC-K)

Since the beginning of the last century, chronic diseases have progressively replaced infectious diseases in terms of their prevalence and impact on human health. Caring for people with chronic conditions has emerged as one of the major challenges facing health care systems, which remain rooted in episodic and acute care. The world- wide aging phenomenon, along with individuals' longer survival following formerly fatal events, are the main drivers of the increasing burden of chronic diseases. As a consequence, the prevalence of multimorbidity, defined as the coexistence of two or more chronic diseases in the same person, is as high as $90 \%$ in older adults (1). 
Despite the increasing number of studies on the occurrence of multimorbidity across age, gender, and socioeconomic strata, its epidemiology remains poorly understood. In fact, given the wide heterogeneity of people suffering from multimorbidity, no single definition or operationalization seems to serve both research and clinical purposes effectively. For example, the exclusive use of a quantitative approach (ie, the number of co-occurring chronic diseases) fails to capture the clustering of chronic diseases in patterns of multimorbidity (2). Studies attempting to describe multimorbidity patterns have used different methodological approaches to address this issue, such as estimation of observed to expected ratios or odds ratios among the most commonly coexisting dyads or triads of chronic conditions, or cluster and factor analyses to identify systematic groupings among diseases. However, these statistical techniques limit interpretation of results and clinical applicability in, for example, their need for large samples, multiple comparisons, overestimation of effect sizes, and the forcing of diseases into single clusters according to similarity or dissimilarity measures. Moreover, previous studies have focused on identifying patterns of diseases rather than clusters of individuals (3), which has prevented researchers from characterizing such patterns in terms of their clinical and social significance. Although some studies have described multimorbidity patterns in terms of their associated burden of polypharmacy (4) or hospital care (5), other individual-level characteristics such as sociodemographic, lifestyle, clinical, and physical and cognitive functional measures have not yet been explored.

In the present study, we aim to build on previous work by applying a soft clustering technique (ie, the fuzzy $c$-means cluster algorithm) to analyze patterns of multimorbidity in a population-based Swedish cohort study of older people. Soft techniques (c-means) present the following advantages over the hard clustering algorithms (in other words, hierarchical clustering, $k$-means) predominantly used in past studies. First, individuals, and not diseases, are grouped in clusters according to their commonly co-occurring diseases. Second, instead of forcing individuals to belong to one specific cluster, participants are assigned a probability of membership in all identified clusters, which makes more sense from a biological perspective. Finally, one disease can characterize more than one cluster, which allows us to build patterns of multimorbidity that take all possible disease combinations into account $(6,7)$. In summary, by using soft clustering techniques, we place individuals and not their diseases at the center of our analyses (8).

The specific objectives of our study are (i) to identify clusters of older people based on their multimorbidity patterns and (ii) to analyze differences among clusters according to sociodemographic, lifestyle, clinical, and functional characteristics.

\section{Methods}

\section{Study Population}

We used baseline data from the population-based Swedish National Study on Aging and Care in Kungsholmen (SNAC-K) (9). This study consists of community-dwelling and institutionalized older adults aged 60 years and older. Of people born between 1898 and 1943, living in the Kungsholmen district of Stockholm (Sweden), a random sample from 11 age cohorts was invited to participate in the study. Those who accepted were evaluated between 2001 and 2004 for the first time and subsequently followed up every 6 years (those aged $<78$ years) or every 3 years (those aged $\geq 78$ years). At baseline, 3,363 people were examined (participation rate, $73 \%$ ). In our study, 432 participants were excluded because they did not fulfill the inclusion criteria of having multimorbidity (ie, two or more chronic diseases) at baseline. As expected, those excluded were younger, more educated, and less likely to be female than those included in the study $(p<.001)$.

\section{Study Variables}

At each study wave, SNAC-K participants undergo a comprehensive clinical and functional assessment by trained physicians, nurses, and neuropsychologists. Physicians collect information on diagnoses via physical examination, medical history, examination of medical charts, self-reported information, and/or proxy interviews. Clinical parameters, lab tests, medication, and inpatient and outpatient care data are also used to identify specific conditions. All diagnoses are coded according to the International Classification of Diseases 10th revision (ICD-10) and classified into 60 chronic disease categories in accordance with a clinically driven methodology (1). Diseases with a prevalence of $<2 \%$ were excluded to avoid statistical noise and therefore spurious findings in the models. Drugs are coded in accordance with the Anatomical Therapeutic Chemical (ATC) classification.

Participants' demographics (ie, age, sex, education, occupation, living arrangement, and civil status) and lifestyle factors are collected during nurse interviews. Educational attainment was categorized as elementary, high school, and university or higher; main occupation was categorized as manual or non-manual based on the longest job held during the person's lifetime. Civil status was categorized as unmarried, married, divorced, and widowed; smoking was categorized as never, former, and current; and alcohol consumption was categorized as never/occasional, light/moderate, and heavy. Following the recommendations of the World Health Organization (WHO) and the American College of Sports Medicine (ACSM), participants were categorized in three different groups according to the intensity of their physical activity: inadequate (less than or equal to two to three times per month of light and/or moderate/intense exercise), healthenhancing (light exercise several times per week or every day), and fitness-enhancing (moderate/intense exercise several times per week or every day) $(10,11)$.

Life satisfaction was measured using the self-reported index developed by Neugarten and colleagues (12) (LSI-A), which captures five components: zest versus apathy, resolution and fortitude, congruence between desired and achieved goals, positive self-concept, and mood. The LSI-A consists of 12 positive and 8 negative items; in this study, the negative items were reversed and the final scores transformed to a $0-100$ scale with higher values indicating greater life satisfaction (13). The social network index combined indicators of self-reported social connections and social support according to the procedure adopted in the National Social Life, Health, and Aging Project (NSHAP Study) (14) and was subsequently categorized into tertiles labeled as poor, moderate, or rich (15). Self-rated health was assessed by asking participants; "In general, how would you describe your health?" and categorized as very good/excellent and good/poor.

Level of disability was measured as the number of basic activities of daily living (bathing, dressing, toileting, continence, transferring, and eating) and instrumental activities of daily living (grocery shopping, meal preparation, housekeeping, laundry, managing money, using the telephone, taking medications, and using public transportation) a person was unable to perform independently. People living in institutions were assumed to depend on others for grocery shopping, meal preparation, housekeeping, and laundry. Balance was measured as the time (in seconds) a participant could stand on one 
leg (up to 60 seconds). Grip strength was measured with a dynamometer and converted to kilograms. Participants were seated with their arm resting on a table and their elbow flexed at 90 degrees. Walking speed was assessed by asking participants to walk $6 \mathrm{~m}$, or $2.44 \mathrm{~m}$ if the participant reported walking slowly. If the participant was unable to walk or attempted unsuccessfully to walk, a value of 0 was recorded. Cognitive status was assessed by physicians with the Mini-Mental State Examination, which ranges from 30 to 0 (from best to worst possible score). Participants' serum albumin $(\mathrm{g} / \mathrm{L})$, creatinine $(\mu \mathrm{mol} / \mathrm{L})$, and C-reactive protein $(\mathrm{mmol} / \mathrm{L})$ levels were measured at Karolinska Institutet's laboratory following standard procedures.

\section{Statistical Analysis}

Multimorbidity patterns were identified using the fuzzy c-means cluster analysis algorithm, which belongs to the family of soft clustering algorithms. The algorithm estimates $c$ cluster centers (similar to $k$-means) but with fuzziness, so that individuals may belong to more than one pattern. We used the technique to obtain clusters of individuals as well as a membership matrix that indicated the degree of participation of each subject in each cluster. Through dimensionality reduction (that is, multiple correspondence analysis) we then obtained the input data for the clustering of participants. To determine the number of retained dimensions, the Karlis-SaportaSpinaki rule was used (16). Different degrees of fuzzification and several validation indices were considered to estimate the optimal number of clusters (7). Given the stochastic nature of the clusters, we ran 100 independent clustering repetitions to obtain the average final solution. The consistency and significance of the final solution was evaluated based on clinical criteria. For cross-validation of the model, we randomly sorted individuals into two independent data sets and compared their validation indices. Indices were computed and averaged over 100 repetitions.

To examine the disease patterns characterizing each cluster, observed/expected ratios were calculated by dividing the prevalence of a given disease within a cluster by its prevalence in the overall population. Disease exclusivity, defined as the fraction of participants with the disease included in the cluster over the total number of participants with the disease, was also calculated. A disease was considered to be associated with a given cluster when the observed/ expected ratio was $\geq 2$ or the exclusivity was $\geq 25 \%(17,18)$. The clusters were further compared for the distribution of sociodemographic, lifestyle, clinical, and functional variables using analysis of variance and chi-square tests. Statistical analyses were performed using $\mathrm{R}$ 3.5.1 and Stata 15.

\section{Results}

The study population consisted of 2,931 individuals. The participants' mean age was 76.1 years, and $66.6 \%$ were female. Six point five percent were living in a nursing home. The mean number of chronic condition was 4.5 , and the mean number of drugs was 4.4 . A total of 39 chronic disease categories had a prevalence of $\geq 2 \%$ and were included in the cluster analyses (Table 1).

The following multimorbidity patterns were detected in our population: psychiatric and respiratory diseases (PSY-RESP) $5.4 \%$, heart diseases (HEART) 9.3\%, eye diseases and cancer (EYECANCER) $10.7 \%$, cognitive and sensory impairments (CNS-IMP) $10.6 \%$, and respiratory and musculoskeletal diseases (RESP-MSK) $15.7 \%$. Around half of the study population $(48.4 \%)$ could not be classified into any of the abovementioned patterns but constituted a cluster where nonspecific chronic conditions were over-represented, and which was named UNSPECIFIC. A clinical description of the patterns in terms of diseases with the highest observed/expected ratio and exclusivity values is reported in Supplementary Table S1. In addition, Figure 1 depicts all diseases with observed/expected ratios $\geq 2$ and/or exclusivity values $\geq 25 \%$ in each cluster. The PSYRESP cluster included individuals with neurotic, stress-related and somatoform disorders, depression, sleep disorders (both insomnia and obstructive sleep apnea), and other unspecified neurological and psychiatric conditions; it also included asthma. The HEART cluster included several cardiac diseases along with cerebrovascular disease, diabetes, migraine, and inflammatory arthropathies. The EYE-CANCER cluster included several eye impairments and solid cancers. The CNS-IMP cluster included dementia, psychiatric and cerebrovascular diseases, and visual and hearing problems. The RESP-MSK cluster included the two most frequent respiratory diseases (ie, asthma and chronic obstructive pulmonary disease) and obstructive sleep apnea.

In Table 2, the five clusters are compared with the UNSPECIFIC one in terms of sociodemographic, lifestyle, clinical, and functional characteristics. The PSY-RESP cluster was associated with higher values of alcoholism and neuroticism. The HEART cluster grouped people with the highest number of co-occurring chronic diseases and drug use and the highest levels of serum creatinine and C-reactive protein. Individuals in the EYE-CANCER cluster exhibited the lowest muscle strength. The CNS-IMP cluster grouped people of very old ages who lived in nursing homes and had the lowest physical functional status. Finally, the part of the population not classified in any specific cluster included the youngest people with the lowest mean number of chronic diseases, the best functional and cognitive and status, and the highest life satisfaction.

\section{Discussion}

In the present study, around half of a Swedish cohort of older adults could be classified into five clinically meaningful clusters, named psychiatric and respiratory diseases (PSY-RESP), heart diseases (HEART), respiratory and musculoskeletal diseases (RESP-MSK), cognitive and sensory impairments (CNS-IMP), and eye diseases and cancer (EYE-CANCER). These clusters showed significantly different sociodemographic, lifestyle, clinical, and functional profiles. The other half of the study population was grouped in an unspecific cluster characterized by being younger, having lower numbers of co-occurring diseases and drug use, and good functional and cognitive abilities.

The PSY-RESP cluster included people with asthma along with psychiatric conditions. The co-occurrence of these diseases could be a result of chronic drug treatment with steroids, which can increase neuroticism, depression, and sleep disorders (19). Besides, asthma symptoms have been associated with depression, even in older participants (20). This cluster grouped relatively young people with alcohol abuse problems and low life satisfaction. The association between alcohol use and psychiatric disorders is well known (21), and this study confirms such an association in older persons. Poor quality of life in people affected by psychiatric and respiratory disorders has also been reported previously $(22,23)$.

The HEART cluster illustrates the well-known link between cardio- and cerebrovascular diseases; atrial fibrillation and heart failure are both risk factors for stroke (24), and diabetes is a risk 
Table 1. Disease Prevalence at Baseline in the Swedish National Study on Aging and Care in Kungsholmen $(N=2,931)$

\begin{tabular}{|c|c|c|}
\hline Rank & Chronic Conditions & Prevalence $(\%)$ \\
\hline 1 & Hypertension & 73.29 \\
\hline 2 & Dyslipidemia & 50.12 \\
\hline 3 & Chronic kidney diseases & 37.84 \\
\hline 4 & Ischemic heart disease & 17.50 \\
\hline 5 & Colitis and related diseases & 14.43 \\
\hline 6 & Osteoarthritis and other degenerative joint diseases & 14.23 \\
\hline 7 & Anemia & 13.68 \\
\hline 8 & Deafness, hearing impairment & 13.07 \\
\hline 9 & Obesity & 13.07 \\
\hline 10 & Heart failure & 12.04 \\
\hline 11 & Thyroid diseases & 11.84 \\
\hline 12 & Atrial fibrillation & 11.02 \\
\hline 13 & Dementia & 10.85 \\
\hline 14 & Depression and mood diseases & 10.44 \\
\hline 15 & Solid neoplasm & 10.10 \\
\hline 16 & Diabetes & 9.96 \\
\hline 17 & Cerebrovascular disease & 8.94 \\
\hline 18 & Osteoporosis & 7.68 \\
\hline 19 & Other musculoskeletal and joint diseases & 7.44 \\
\hline 20 & Dorsopathies & 7.30 \\
\hline 21 & Asthma & 6.86 \\
\hline 22 & Glaucoma & 6.38 \\
\hline 23 & Cataract and other lens diseases & 6.24 \\
\hline 24 & Other eye diseases & 5.70 \\
\hline 25 & Chronic obstructive pulmonary disease, emphysema, chronic bronchitis & 5.66 \\
\hline 26 & Autoimmune diseases & 5.12 \\
\hline 27 & Esophagus, stomach, and duodenum diseases & 4.95 \\
\hline 28 & Blindness, visual impairment & 4.91 \\
\hline 29 & Inflammatory arthropathies & 4.57 \\
\hline 30 & Prostate diseases & 4.50 \\
\hline 31 & Other cardiovascular diseases & 3.92 \\
\hline 32 & Neurotic, stress-related, and somatoform diseases & 3.55 \\
\hline 33 & Other genitourinary diseases & 2.87 \\
\hline 34 & Cardiac valve diseases & 2.83 \\
\hline 35 & Migraine and facial pain syndromes & 2.59 \\
\hline 36 & Other psychiatric and behavioral diseases & 2.52 \\
\hline 37 & Sleep disorders & 2.39 \\
\hline 38 & Other neurological diseases & 2.18 \\
\hline 39 & Bradycardias and conduction diseases & 2.12 \\
\hline
\end{tabular}

factor for stroke and coronary heart disease (25). The high prevalence of migraine in this cluster may be explained by either brain vascular pathology or by the drugs prescribed for cardiac diseases, such as nitrates $(26,27)$. The high number of co-occurring chronic conditions and drugs were distinctive of individuals belonging to this cluster. This cluster had the second highest percentage of persons, after the CNS-IMP cluster, with limitations in activities of daily living and instrumental activities of daily living. Individuals in this cluster also showed the highest serum creatinine and C-reactive protein levels. Expression of proinflammatory cytokines increases throughout the human life span, and this increase is correlated with cardiovascular health (28). Chronic low-grade inflammation, in turn, promotes autonomic imbalance, stimulates remodeling, depresses cardiac function, prompts endothelial dysfunction, and leads to a progression of atherosclerosis and impaired renal function (29).

The RESP-MSK cluster included osteoporosis possibly related to the chronic treatment of asthma and chronic obstructive pulmonary disease with steroids (30). Vitamin D deficiency could also underlie both respiratory and skeletal disorders; vitamin D supplements are beneficial both in preventing exacerbations of chronic obstructive pulmonary disease and improving bone density measures (31,32). The presence of upper gastrointestinal system disorders can also be related to the treatment of respiratory diseases and the use of diphosphonates in osteoporosis (33), whereas thyroid and other autoimmune diseases are often correlated (34).

The EYE-CANCER cluster included several eye impairments and solid cancers. A high percentage of participants in this cluster were widowed, which is explained by their higher age. Old age may also explain why they have the lowest grip strength.

The CNS-IMP cluster brings to light the association recently found between sensorial impairment and dementia. Hearing deficits have attracted much interest, motivated by strong evidence that impaired hearing is a risk factor for cognitive decline and dementia $(35,36)$. The relationship between vision loss and dementia has been evaluated in cross-sectional and longitudinal studies; the 3C cohort study suggested that poor vision, in particular near vision loss, may be an indicator of dementia risk at short and middle term (37). Retinal microvasculature pathology has been associated with vascular dementia, especially in persons with diabetes (38). Multiple sensorial impairments have also been found to increase dementia 


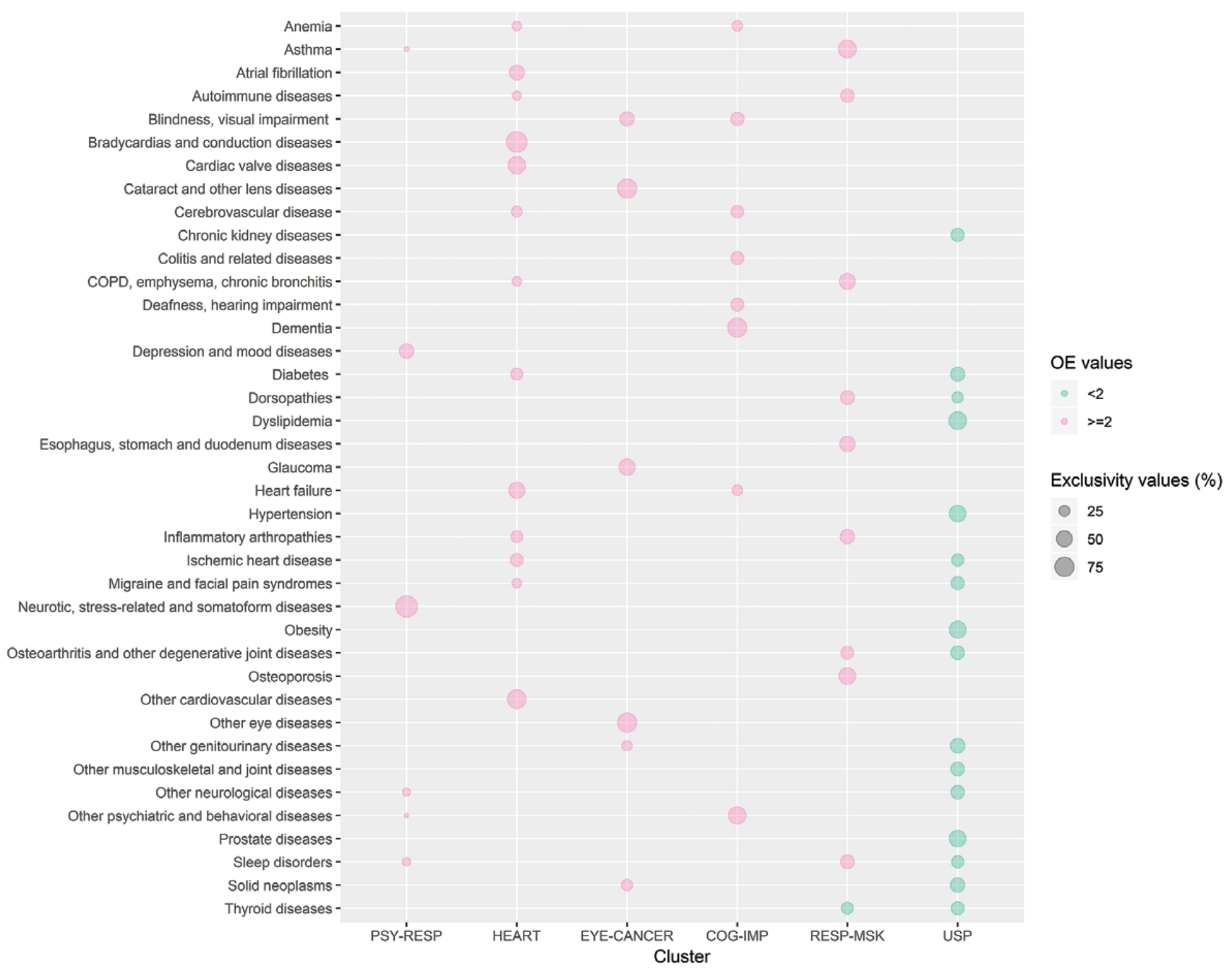

Figure 1. Chronic diseases characterizing clusters of older people identified at baseline in the Swedish National Study on Aging and Care in Kungsholmen ( $N=2$,931). PSY-RESP: psychiatric and respiratory diseases; HEART: heart diseases; EYE-CANCER: eye diseases and cancer; CNS-IMP: cognitive and sensory impairments; RESP-MSK: respiratory and musculoskeletal diseases; O/E ratio: observed/expected ratio.

risk (39). Persons in this cluster were very old and had the worst levels of physical and cognitive function; this justifies why $43 \%$ of them were living in a nursing home. Any disease in the cluster could explain the functional impairment, particularly dementia and cerebrovascular diseases (40). This cluster grouped the highest percentage of people who were manual workers with low education. Low educational attainment and a manual occupation during early life have been consistently associated with an increased risk of dementia (41) and poor income in later life. Finally, this cluster was also characterized by having the highest percentage of persons with a poor social network and inadequate physical activity levels.

\section{Clinical and Public Health Implications}

Despite the high prevalence of multimorbidity in the older population, knowledge about how chronic diseases co-occur in single individuals is limited. Furthermore, findings from different studies are hardly comparable with the literature in the field because of differing methodological approaches.

In a previous study from an older Swedish population, the coexistence of diseases was evaluated with a cluster analysis approach (2).
Cluster analysis groups diseases according to their similarity forcing each disease to be part of one single cluster; this approach can be particularly useful to generate new research hypotheses on the pathophysiological correlations as well as the strength of causal associations between diseases. Conversely, the soft technique employed in the present study has the main advantage to group individuals who, according to their commonly co-occurring diseases, belong to different multimorbidity patterns, enabling one same disease to belong to more than one cluster. In addition, individuals are provided with a probability to belong to each of the identified clusters, and the most probable membership was investigated in our analyses. This methodology can be advantageous to describe the overall health status of a specific population, providing particularly useful information from a clinical point of view.

First, groups of people at high risk of adverse health outcomes can be identified and may benefit from targeted secondary and tertiary preventative interventions. For example, individuals in the HEART cluster may develop disabilities for a number of reasons, such as dyspnea in heart failure, stroke sequelae, and/or peripheral atherosclerosis and neuropathy from diabetes. Yet a correct identification of the exact cause of the functional limitations could be particularly important to this group of individuals to plan correct measures and 
Table 2. Sociodemographic, Lifestyle, Clinical, and Functional Differences Among Clusters of Older People at Baseline in Swedish National Study on Aging and Care in Kungsholmen $(N=2,931)$

\begin{tabular}{|c|c|c|c|c|c|c|c|}
\hline & PSY-RESP & HEART & EYE-CANCER & CNS-IMP & RESP-MSK & UNSPECIFIC & ALL \\
\hline$n(\%)$ & $159(5.4)$ & $272(9.3)$ & $313(10.7)$ & $309(10.6)$ & $460(15.7)$ & $1,418(48.4)$ & 2,931 \\
\hline \multicolumn{8}{|l|}{ Sociodemographic factors } \\
\hline Female sex (\%) & 74.2 & 59.2 & 72.5 & 76.9 & 74.4 & 61.0 & 66.6 \\
\hline Age, mean & 73.3 & 82.3 & 83.2 & 88.2 & 73.9 & 71.8 & 76.1 \\
\hline Living in a nursing home $(\%)$ & 5.8 & 6.0 & 4.1 & 43.2 & 1.8 & 0.7 & 6.5 \\
\hline \multicolumn{8}{|l|}{ Civil status $(\%)$} \\
\hline Unmarried & 17.0 & 16.2 & 16.1 & 21.1 & 17.8 & 16.4 & 17.1 \\
\hline Married & 35.2 & 35.5 & 25.1 & 25.8 & 38.4 & 49.5 & 40.6 \\
\hline Divorced & 20.9 & 8.2 & 11.2 & 7.5 & 16.8 & 13.0 & 12.8 \\
\hline Widow & 26.9 & 40.1 & 47.6 & 45.6 & 27.0 & 21.1 & 29.5 \\
\hline \multicolumn{8}{|l|}{ Education (\%) } \\
\hline Elementary & 16.9 & 25.1 & 21.8 & 34.8 & 19.3 & 14.4 & 19.1 \\
\hline High school & 47.6 & 56.1 & 56.5 & 47.4 & 49.5 & 50.1 & 50.9 \\
\hline University & 35.5 & 18.8 & 21.8 & 17.7 & 31.1 & 35.5 & 30.0 \\
\hline \multicolumn{8}{|l|}{ Occupation $(\%)$} \\
\hline Manual worker & 19.1 & 31.7 & 33.0 & 39.8 & 24.0 & 21.5 & 25.6 \\
\hline Non-manual worker & 80.9 & 68.3 & 67.0 & 60.2 & 76.0 & 78.5 & 74.4 \\
\hline Life satisfaction score, mean & 46.5 & 49.6 & 53.3 & 48.2 & 57.3 & 60.1 & 57.1 \\
\hline \multicolumn{8}{|l|}{ Social network $(\%)$} \\
\hline Poor & 39.7 & 50.1 & 43.2 & 67.7 & 31.8 & 27.0 & 34.9 \\
\hline Moderate & 35.8 & 24.4 & 36.5 & 21.3 & 35.4 & 35.8 & 33.8 \\
\hline Rich & 24.5 & 25.5 & 20.3 & 11.0 & 32.9 & 37.2 & 31.2 \\
\hline \multicolumn{8}{|l|}{ Lifestyle factors } \\
\hline \multicolumn{8}{|l|}{ Smoking (\%) } \\
\hline Never & 43.6 & 45.4 & 59.2 & 60.1 & 45.5 & 46.7 & 48.7 \\
\hline Former & 35.1 & 44.1 & 31.2 & 30.9 & 39.7 & 38.3 & 37.5 \\
\hline Current & 21.3 & 10.6 & 9.6 & 9.0 & 14.8 & 15.0 & 13.8 \\
\hline \multicolumn{8}{|l|}{ Alcohol consumption $(\%)$} \\
\hline Never/occasional & 39.7 & 52.7 & 51.2 & 77.6 & 40.4 & 30.1 & 40.8 \\
\hline Light/moderate & 36.9 & 37.2 & 35.0 & 17.3 & 42.1 & 53.1 & 43.8 \\
\hline Heavy & 23.4 & 10.1 & 13.8 & 5.1 & 17.5 & 16.9 & 15.3 \\
\hline \multicolumn{8}{|l|}{ Physical activity (\%) } \\
\hline Inadequate & 42.4 & 56.0 & 44.7 & 82.9 & 31.1 & 22.7 & 36.9 \\
\hline Health-enhancing & 40.2 & 36.3 & 45.8 & 14.4 & 50.1 & 52.5 & 45.2 \\
\hline Fitness-enhancing & 17.4 & 7.7 & 9.6 & 2.6 & 18.8 & 24.7 & 17.9 \\
\hline \multicolumn{8}{|l|}{ Clinical and functional factors } \\
\hline \multicolumn{8}{|l|}{ Self-rated health (\%) } \\
\hline Very good/excellent & 15.4 & 5.1 & 23.0 & 14.8 & 23.6 & 43.3 & 32.5 \\
\hline Good/poor & 84.6 & 94.9 & 77.0 & 85.2 & 76.4 & 56.7 & 67.5 \\
\hline Chronic conditions, mean & 5.7 & 7.7 & 6.0 & 5.5 & 4.7 & 3.2 & 4.5 \\
\hline Drugs, mean & 6.2 & 7.7 & 5.0 & 6.1 & 5.3 & 2.8 & 4.4 \\
\hline Serum albumin $(\mathrm{g} / \mathrm{L})$, mean & 41.5 & 40.5 & 40.0 & 38.7 & 40.9 & 42.0 & 41.2 \\
\hline Serum creatinine (umol/L), mean & 86.1 & 107.5 & 95.8 & 98.6 & 87.7 & 87.3 & 90.9 \\
\hline Serum CRP $(\mathrm{mmol} / \mathrm{L})$, mean & 6.5 & 8.7 & 6.9 & 8.6 & 7.3 & 6.1 & 6.8 \\
\hline ADL + IADL limitations, mean & 1.3 & 2.1 & 1.4 & 7.2 & 0.7 & 0.3 & 1.4 \\
\hline Balance test (s), mean & 22.9 & 9.2 & 9.6 & 4.3 & 22.7 & 30.1 & 22.6 \\
\hline Grip strength test $(\mathrm{N})$, mean & 22.2 & 22.7 & 20.3 & 23.7 & 22.2 & 26.7 & 24.7 \\
\hline Walking speed $(\mathrm{m} / \mathrm{s})$, mean & 0.9 & 0.6 & 0.7 & 0.3 & 0.9 & 1.1 & 0.9 \\
\hline MMSE test, mean & 27.7 & 27.6 & 27.7 & 16.6 & 28.6 & 28.8 & 27.4 \\
\hline
\end{tabular}

Notes: PSY-RESP = psychiatric and respiratory diseases; HEART = heart diseases; EYE-CANCER = eye diseases and cancer; CNS-IMP = cognitive and sensory impairments; RESP-MSK = respiratory and musculoskeletal diseases; CRP = C-reactive protein; ADL = activities of daily living; IADL = instrumental activities of daily living; MMSE = Mini-Mental State Examination. Missing values (\%): age (0.3), civil status (0.5), education (1.1), occupation (2.7), life satisfaction score (39.2), social network (11.6), smoking (3.3), alcohol consumption (3.2), self-rated health (31.4), drugs (0.2), serum albumin (8.6), serum creatinine (8.6), serum CRP (10.0), ADL + IADL limitations (3.8), balance test (10.4), grip strength (25.1), walking speed (4.0), MMSE test (6.8). The distribution of all variables was significantly different across clusters $(p<.001)$.

prevent disability. People in the CNS-IMP cluster could be systematically screened for functional impairment; if present, physical rehabilitation could be prescribed that might delay the progression to disability. Although no improvement has been shown in cognitive functions, there is promising evidence that exercise programs may improve the ability to perform activities of daily living for people with dementia (42). People in the PSY-RESP cluster may benefit from specific interventions designed to reduce alcohol abuse and 
subsequently improve their quality of life. Individuals in the EYECANCER cluster, characterized by a low muscle strength, may be screened for sarcopenia, given the known association between low muscle mass and chemotoxicity (43).

Second, care management could be improved for people with specific patterns characterized, for example, by polypharmacy and therefore a high frequency of potentially inappropriate medication and adverse drug reactions (44). Recent guidelines specifically developed for people with multimorbidity underline the potential treatment burden for patients prescribed a high number of drugs (45), such as those in the HEART cluster. In fact, certain therapeutic regimens that are appropriate for diseases affecting people in middle adulthood could be associated with the development of specific patterns of co-occurring diseases in late life. Some examples are chronic steroid treatment for respiratory diseases and the development of skeletal and psychiatric disorders (30), or chronic treatment with anticholinergic drugs for psychiatric diseases and the development of dementia in older age (46).

Finally, the group of people included in the UNSPECIFIC cluster is particularly interesting from both a research and prevention point of view. In fact, people in this pattern, despite suffering from two or more chronic diseases, were relatively younger, suggesting that aging itself is the main driver of disease clustering. This finding strengthens the idea that aging and multimorbidity share pathophysiological mechanisms (28) and that their connection is more evident when we analyze not only the number but also the patterns of diseases. Furthermore, the identification of this group of people is fundamental to plan interventions for the primary prevention of disease accumulation and to distribute health care resources accordingly.

\section{Strengths and Limitations}

The main strength of this study is the statistical technique, applied to allow clustering individuals according to their co-occurring diseases. The fuzzy $c$-means cluster algorithm is used for pattern recognition when clusters tend to overlap, which is most often the case in older adults. Other strengths are the high number of very old people in the cohort and the comprehensive list of both mental and physical chronic conditions included in the analyses. Limitations include the cross-sectional design of the study and the average high socioeconomic status of participants in SNAC-K, which limits the external validity of the findings.

\section{Conclusion}

In the present study, half of a cohort of older adults could be classified into five clinically meaningful clusters. These clusters showed significantly different sociodemographic, lifestyle, clinical, and functional profiles. This and similar approaches to the epidemiological study of multimorbidity are needed, not only to better understand the complex interactions among co-occurring diseases but also, even more importantly, to improve preventive interventions and optimally address individuals' care needs and the risk of adverse outcomes.

\section{Supplementary Material}

Supplementary data are available at The Journals of Gerontology, Series A: Biological Sciences and Medical Sciences online.

\section{Funding}

This work was supported by the founders of the Swedish National study on Aging and Care (SNAC): the Italian Ministry of Health (PE-2016-02364885); the Ministry of Health and Social Affairs, Sweden; the participating county councils and municipalities; and the Swedish Research Council. Specific grants were obtained from the Swedish Research Council (2016-00981), the Swedish Research Council for Health, Working Life and Welfare (2017-01764).

\section{Acknowledgments}

We thank the SNAC-K participants and the SNAC-K group for their collaboration in data collection and management. The study was approved by the Regional Ethics Review Board in Stockholm. Participants in the study completed a written informed consent form as stipulated by the ethics board. For participants with prevalent or incident cognitive impairment, consent was obtained from next of kin.

\section{Author Contributions}

A.R.L., D.L.V., and A.C.L. developed the study concept and design. A.R.L. performed the data analysis, and A.M., D.L.V., and A.C.L. contributed to the interpretation of the results. A.M. and A.R.L. drafted the manuscript. All authors provided critical revisions and approved the final version of the manuscript for submission.

\section{Conflict of Interest}

None reported.

\section{References}

1. Calderón-Larrañaga A, Vetrano DL, Onder G, et al. Assessing and measuring chronic multimorbidity in the older population: a proposal for its operationalization. J Gerontol A Biol Sci Med Sci. 2017;72:1417-1423. doi:10.1093/gerona/glw233

2. Marengoni A, Rizzuto D, Wang HX, Winblad B, Fratiglioni L. Patterns of chronic multimorbidity in the elderly population. J Am Geriatr Soc. 2009;57:225-230. doi:10.1111/j.1532-5415.2008.02109.x

3. Prados-Torres A, Calderón-Larrañaga A, Hancco-Saavedra J, PobladorPlou B, van den Akker M. Multimorbidity patterns: a systematic review. J Clin Epidemiol. 2014;67:254-266. doi:10.1016/j.jclinepi.2013.09.021

4. Nobili A, Marengoni A, Tettamanti M, et al. Association between clusters of diseases and polypharmacy in hospitalized elderly patients: results from the REPOSI study. Eur J Intern Med. 2011;22:597-602. doi:10.1016/j. ejim.2011.08.029

5. Collerton J, Jagger C, Yadegarfar ME, et al. Deconstructing complex multimorbidity in the very old: findings from the Newcastle $85+$ Study. Biomed Res Int. 2016;2016:8745670. doi:10.1155/2016/8745670

6. Everitt B. Cluster Analysis. 5th ed. Chichester, UK: Wiley; 2011.

7. Zhao Q. Cluster Validity in Clustering Methods [dissertation]. Joensuu, Finland: University of Eastern Finland; 2012:87. http://cs.joensuu.fi/sipu/ pub/qinpei-thesis.pdf. Accessed June 4, 2019.

8. Capobianco E, Lio' P. Comorbidity: a multidimensional approach. Trends Mol Med. 2013;19:515-521. doi:10.1016/j.molmed.2013.07.004

9. Lagergren M, Fratiglioni L, Hallberg IR, et al. A longitudinal study integrating population, care and social services data. The Swedish National Study on Aging and Care (SNAC). Aging Clin Exp Res. 2004;16:158-168

10. Rydwik E, Welmer AK, Kareholt I, et al. Adherence to physical exercise recommendations in people over 65-the SNAC-Kungsholmen study. Eur J Public Health 2013;23:799-804. doi: 10.1093/eurpub/cks150

11. Nelson ME, Rejeski WJ, Blair SN, et al. Physical activity and public health in older adults: recommendation from the American College of Sports Medicine and the American Heart Association. Med Sci Sports Exerc. 2007;39:1435-1445. doi:10.1249/mss.0b013e3180616aa2

12. Neugarten BL, Havighurst RJ, Tobin SS. The measurement of life satisfaction. J Gerontol. 1961;16:134-143.

13. Okun MA, Stock WA. The construct validity of subjective well-being measures: an assessment via quantitative research syntheses. J Community Psychol. 1987; 15:481-492. 
14. Cornwell EY, Waite LJ. Measuring social isolation among older adults using multiple indicators from the NSHAP study. J Gerontol B Psychol Sci Soc Sci. 2009; 64(suppl 1):i38-i46. doi:10.1093/geronb/gbp037

15. Calderón-Larrañaga A, Santoni G, Wang HX, et al. Rapidly developing multimorbidity and disability in older adults: does social background matter? J Intern Med. 2018;283:489-499. doi:10.1111/joim.12739

16. Sourial N, Wolfson C, Zhu B, et al. Correspondence analysis is a useful tool to uncover the relationships among categorical variables. J Clin Epidemiol. 2010;63:638-646. doi:10.1016/j.jclinepi.2009.08.008

17. Guisado-Clavero M, Roso-Llorach A, López-Jimenez T, et al. Multimorbidity patterns in the elderly: a prospective cohort study with cluster analysis. BMC Geriatr. 2018;18:16. doi:10.1186/ s12877-018-0705-7

18. Violán C, Roso-Llorach A, Foguet-Boreu Q, et al. Multimorbidity patterns with K-means nonhierarchical cluster analysis. BMC Fam Pract. 2018;19:108. doi:10.1186/s12875-018-0790-x

19. Lebedeva KA, Caruncho HJ, Kalynchuk LE. Cyclical corticosterone administration sensitizes depression-like behavior in rats. Neurosci Lett. 2017;650:45-51. doi:10.1016/j.neulet.2017.04.023

20. de Roos EW, Lahousse L, Verhamme KMC, et al. Asthma and its comorbidities in middle-aged and older adults: the Rotterdam Study. Respir Med. 2018;139:6-12. doi:10.1016/j.rmed.2018.04.006

21. Carton L, Pignon B, Baguet A, et al. Influence of comorbid alcohol use disorders on the clinical patterns of major depressive disorder: a general population-based study. Drug Alcohol Depend. 2018;187:40-47. doi:10.1016/j.drugalcdep.2018.02.009

22. Boyer L, Baumstarck K, Boucekine M, Blanc J, Lançon C, Auquier P. Measuring quality of life in patients with schizophrenia: an overview. Expert Rev Pharmacoecon Outcomes Res. 2013;13:343-349. doi:10.1586/erp.13.15

23. Oga T, Windisch W, Handa T, Hirai T, Chin K. Health-related quality of life measurement in patients with chronic respiratory failure. Respir Investig. 2018;56:214-221. doi:10.1016/j.resinv.2018.01.006

24. Haeusler KG, Gröschel K, Köhrmann M, et al. Expert opinion paper on atrial fibrillation detection after ischemic stroke. Clin Res Cardiol. 2018;107:871-880. doi:10.1007/s00392-018-1256-9

25. Mitsios JP, Ekinci EI, Mitsios GP, Churilov L, Thijs V. Relationship between glycated hemoglobin and stroke risk: a systematic review and meta-analysis. J Am Heart Assoc. 2018;7:e007858. doi:10.1161/ JAHA.117.007858

26. Bektas F, Soyuncu S. Nitroglycerin-induced migraine type headache: bilaterally visible temporal arteries. Int J Emerg Med. 2010;3:463-464. doi:10.1007/s12245-010-0248-y

27. Mason BN, Russo AF. Vascular contributions to migraine: time to Revisit? Front Cell Neurosci. 2018;12:233. doi:10.3389/fncel.2018.00233

28. Ferrucci L, Fabbri E. Inflammageing: chronic inflammation in ageing, cardiovascular disease, and frailty. Nat Rev Cardiol. 2018;15:505-522. doi:10.1038/s41569-018-0064-2

29. Smykiewicz P, Segiet A, Keag M, Żera T. Proinflammatory cytokines and ageing of the cardiovascular-renal system. Mech Ageing Dev. 2018;175:35-45. doi:10.1016/j.mad.2018.07.006

30. Kanis JA, Stevenson M, McCloskey EV, Davis S, Lloyd-Jones M. Glucocorticoid-induced osteoporosis: a systematic review and cost-utility analysis. Health Technol Assess. 2007;11:iii-iv, ix.
31. Zografos GN, Georgiadou D, Thomas D, Kaltsas G, Digalakis M. Drug-induced esophagitis. Dis Esophagus. 2009;22:633-637. doi:10.1111/j.1442-2050.2009.00972.x

32. Jolliffe DA, Greenberg L, Hooper RL, et al. Vitamin D to prevent exacerbations of COPD: systematic review and meta-analysis of individual participant data from randomised controlled trials. Thorax. 2019;74:337345. doi:10.1136/thoraxjnl-2018-212092

33. Shahnazari B, Moghimi J, Foroutan M, Mirmohammadkhani M, Ghorbani A. Comparison of the effect of vitamin D on osteoporosis and osteoporotic patients with healthy individuals referred to the Bone Density Measurement Center. Biomol Concepts. 2019;10:44-50. doi:10.1515/bmc-2019-0005

34. Luo W, Mao P, Zhang L, Yang Z. Association between systemic lupus erythematosus and thyroid dysfunction: a meta-analysis. Lupus. 2018;27:2120-2128. doi:10.1177/0961203318805849

35. Shen Y, Ye B, Chen P, et al. Cognitive decline, dementia, Alzheimer's disease and presbycusis: examination of the possible molecular mechanism. Front Neurosci. 2018;12:394. doi:10.3389/fnins.2018.00394

36. Livingston G, Sommerlad A, Orgeta V, et al. Dementia prevention, intervention, and care. Lancet. 2017;390:2673-2734. doi:10.1016/ S0140-6736(17)31363-6

37. Naël V, Pérès K, Dartigues JF, et al.; Sense-Cog consortium. Vision loss and 12-year risk of dementia in older adults: the 3C cohort study. Eur J Epidemiol. 2019;34:141-152. doi:10.1007/s10654-018-00478-y

38. Brenowitz WD, Kaup AR, Lin FR, Yaffe K. Multiple sensory impairment is associated with increased risk of dementia among black and white older adults. J Gerontol A Biol Sci Med Sci. 2019;74:890-896. doi:10.1093/ gerona/gly 264

39. Deal JA, Sharrett AR, Albert M, et al. Retinal signs and risk of incident dementia in the Atherosclerosis Risk in Communities study. Alzheimers Dement. 2019;15:477-486. doi:10.1016/j.jalz.2018.10.002

40. Mograbi DC, Morris RG, Fichman HC, et al. The impact of dementia, depression and awareness on activities of daily living in a sample from a middle-income country. Int J Geriatr Psychiatry. 2018;33:807-813. doi:10.1002/gps.4765

41. Marengoni A, Fratiglioni L, Bandinelli S, Ferrucci L. Socioeconomic status during lifetime and cognitive impairment no-dementia in late life: the population-based aging in the Chianti Area (InCHIANTI) Study. J Alzheimers Dis. 2011;24:559-568. doi:10.3233/JAD-2011-101863

42. Forbes D, Thiessen EJ, Blake CM, Forbes SS, Forbes S. Exercise programs for people with dementia. Cochrane Database Syst Rev. 2013; 12: CD006489. doi:10.1002/14651858.CD006489.pub3

43. Wendrich AW, Swartz JE, Bril SI, et al. Low skeletal muscle mass is a predictive factor for chemotherapy dose-limiting toxicity in patients with locally advanced head and neck cancer. Oral Oncol. 2017;71:26-33. doi:10.1016/j.oraloncology.2017.05.012

44. Davies EA, O’Mahony MS. Adverse drug reactions in special populations - the elderly. Br J Clin Pharmacol. 2015;80:796-807. doi:10.1111/ bcp. 12596

45. Farmer C, Fenu E, O'Flynn N, Guthrie B. Clinical assessment and management of multimorbidity: summary of NICE guidance. BMJ. 2016;354:i4843. doi:10.1136/bmj.i4843

46. Richardson K, Fox C, Maidment I, et al. Anticholinergic drugs and risk of dementia: case-control study. BMJ. 2018;361:k1315. doi:10.1136/bmj. k1315 